\title{
EFEITO DE DOIS PROCEDIMENTOS DE ENSINO SOBRE O COMPORTAMENTO DE ORDENAR
}

\author{
Jaci Augusta Neves de Souza* \\ Grauben José Alves de Assis
}

\begin{abstract}
RESUMO. As relações entre estímulos podem ser documentadas por meio de testes comportamentais. O presente estudo teve por objetivo comparar o efeito de duas táticas de ensino sobre o comportamento de ordenar. Doze participantes portadores de necessidades educacionais especiais de ambos os sexos, distribuídos em dois grupos experimentais, foram expostos às duas táticas de ensino. O Grupo A iniciou pelo procedimento de ensino por encadeamento e os participantes do Grupo B pelo procedimento de justaposição. Testes comportamentais avaliaram a produção de seqüências de até cinco estímulos em ordem decrescente. A tarefa dos participantes era ordenar os estímulos. Nove participantes apresentaram desempenhos consistentes com a literatura nos testes, sugerindo a formação de classes ordinais. O ensino por encadeamento mostrou-se mais eficaz. A característica fundamental deste procedimento pode estar na exposição continuada dos estímulos nas tentativas de ensino. A justaposição de estímulos parece induzir a mais erros, provavelmente, pela supressão dos estímulos à medida que novos estímulos são adicionados.
\end{abstract}

Palavras-chave: classes ordinais, sequiências, portadores de necessidades educacionais especiais.

\section{EFFECT OF TWO TEACHING PROCEDURES ON ORDERNESS}

\begin{abstract}
The relationships between the stimuli can be documented by behavioral tests. The objective of the present study was to compare effects of two distinct teaching methods in the formation of ordinal classes. The participants were twelve students with special educational needs, of both sexes, divided in two experimental groups. The participants of Group A began with the chaining method and the participants of Group B with overlapping method. Behavioral tests evaluate the production of sequences of five stimuli. The required response was to select stimuli to form three sequences in decreasing order. Nine participants performed consistently in the tests suggesting formation of the ordinal classes. The chaining method showed effectiveness. The fundamental characteristic can be attributed to continued exposure to stimuli in teaching. The overlapping two stimuli appear to induce to more errors, probably to the suppression of stimuli while new stimuli are being added in the setting.
\end{abstract}

Key words: ordinal classes, sequence, mental retardation. ${ }^{1}$

Segundo Catania (1999), denomina-se aprendizagem verbal o responder a seqüências de letras que se organizam formando sílabas, palavras, frases, orações. Alguns procedimentos podem estabelecer repertórios verbais, entre eles a aprendizagem serial, em que os estímulos verbais são relacionados arbitrariamente em uma ordem específica, determinada pelas contingências de reforçamento. No cotidiano, observam-se vários exemplos de cadeias verbais: recitar um poema, cantar um hino ou ainda rezar uma oração.
A análise do efeito da posição do estímulo em sequiências comportamentais tem sido extensamente investigada em sujeitos não-humanos. Sidman e Rosenberger (1967) publicaram, em um artigo já clássico na literatura, que macacos respondiam, em diferentes chaves de respostas, numa longa seqüência de posições a partir da luminosidade por elas sinalizada, que era esvaecida ${ }^{1}$. O procedimento envolvia discriminação simples com luminosidade sendo sobrepostas. Os participantes respondiam a sequiências de estímulos cada vez mais longas. O

* Mestre em Psicologia pela Universidade Federal do Pará.

\# Doutor em Psicologia. Professor Adjunto do Departamento de Psicologia do Desenvolvimento e da Personalidade da Universidade Federal do Rio Grande do Sul-UFRGS.

1 Fading - um procedimento pelo qual o reforçamento diferencial torna-se contingente à emissão de respostas na presença de mudanças graduais - introdução ou retirada - nas dimensões dos estímulos. 
procedimento de esvanecimento (fading) ${ }^{l}$ também foi usado para estabelecer desempenhos mais complexos para macacos rhesus (Mackay \& Brown, 1971) a partir de seqüências de posição serial.

Táticas de ensino têm sido propostas para estudar o estabelecimento de relações de ordem entre os estímulos dentro e por meio de seqüências. Uma destas táticas é o treino por encadeamento, em que os estímulos são adicionados de forma gradual até que todos os elementos de um conjunto previamente estabelecido sejam apresentados simultaneamente. Outra tática de ensino que tem sido proposta é a justaposição de estímulos, que estabelece várias sequiências de dois estímulos justapostos em vez de apenas uma seqüência que introduz gradativamente novos estímulos (Green, Stromer \& Mackay, 1993).

Stevens (1951) descreveu as seguintes propriedades para estudar essas relações: irreflexividade, assimetria, transitividade e conectividade. Para definir as propriedades, são usados códigos alfanuméricos e o símbolo $\rightarrow$ indicando qual termo vem antes e qual vem depois. A irreflexividade define-se pelo fato de que um estímulo não vem antes dele próprio. Não há nenhum evento na natureza que preceda ou suceda a si mesmo, comportamentalmente. A assimetria mostra que a relação é unidirecional, isto é, se A > B o inverso não é verdadeiro. Uma relação ordinal apresenta transitividade quando pares de estímulos nãoadjacentes em seqüência são relacionados pelo participante, por exemplo, selecionar 2 antes do 4 na seqüência 1, 2, 3, 4, 5, 6. Finalmente, a conectividade é demonstrada quando todas as possibilidades de relações de pares adjacentes e não-adjacentes na seqüência ou entre seqüências diferentes emergem sem qualquer ensino adicional. No mesmo exemplo, selecionar 1 antes de 2 ou antes de 3 ou ainda 4 (numeral) antes de seis (numeral).

Vários estudos experimentais têm buscado compreender como classes ordinais emergem sem qualquer treino adicional a partir de contingências simples de reforçamento (Assis \& Sampaio, 2003; Galy, Camps \& Melan, 2003; Holcomb, Stromer \& Mackay, 1997; Lazar, 1977; Sigurdardottir, Green \& Saunders, 1990; Stromer, Mackay, Cohen \& Stoddard, 1993). Os autores buscam investigar se as funções que os estímulos exercem a partir de suas posições em pelo menos duas sequiências diferentes podem ser substituídas umas pelas outras em novas seqüências, sem qualquer ensino adicional.

Um estudo já considerado clássico na literatura, apresentado por Lazar (1977), sugeriu que os processos comportamentais envolvidos no desenvolvimento de relações de equivalência no contexto de emparelhamento com o modelo também podem estar envolvidos no desenvolvimento do responder seqüencial. $\mathrm{O}$ estudo propôs um delineamento experimental através do qual as classes de equivalência adquirissem novos membros fora do paradigma de emparelhamento de acordo com o modelo. No entanto, as conclusões sobre o estudo devem ser vistas com cautela, pelo fato de que as seqüências envolveram apenas dois estímulos em seqüências de duas posições.

Para dirimir possíveis dúvidas, Sigurdardottir, Green e Saunders (1990) estenderam as classes seqüenciais numa tarefa de três estímulos que incluíam um estímulo de distração (um estímulo que não deveria ser selecionado) e testaram a transferência de funções ordinais para novos membros da classe de equivalência. Os resultados foram consistentes com a literatura.

Green e cols. (1993) descreveram procedimentos para avaliar a natureza das relações entre estímulos em seqüências ensinadas separadamente umas das outras. A tarefa experimental proposta foi ordenar cinco formas abstratas exibidas de maneira gradual até que todas as formas fossem apresentadas. Os estímulos eram exibidos aos pares. Resultados positivos poderiam sugerir que essa tática de ensino estabeleceria uma relação de ordem entre os estímulos consistente com a posição que esses estímulos ocuparam no treino original. Testes poderiam, então, avaliar se os estímulos da mesma posição ordinal a partir de duas ou mais sequiências são substituíveis entre si, formando, portanto, classes de estímulos que ocuparam a mesma posição em sequiências treinadas separadamente.

Nessa mesma direção, Stromer e cols. (1993) investigaram se o treino separado de duas seqüências poderia permitir a produção de novas seqüências misturadas (por exemplo, A1 $\rightarrow \mathrm{B} 2 \rightarrow \mathrm{A} 3 \rightarrow \mathrm{B} 4 \rightarrow \mathrm{A} 5$ ) sem treino direto. Para isso, três adultos e uma criança foram ensinados a produzir uma seqüência de estímulos num procedimento-padrão de encadeamento no qual a tarefa requereu tocar cada uma das cinco diferentes formas abstratas em uma ordem independente da sua localização, de tentativa para tentativa. Nos testes, os participantes produziram imediatamente a seqüência de cinco termos derivada do treino. A contribuição do estudo incluiu o desenvolvimento de novas seqüências de dois termos envolvendo estímulos com posições adjacentes, sem que os autores recorressem a conceitos como representações ou mapas cognitivos.

Esses resultados foram replicados e estendidos por Holcomb e cols. (1997) em três experimentos com crianças normais (entre 3 e 5 anos). O estudo 
objetivou estabelecer as bases para a produção de uma seqüência com seis membros. Os resultados mostraram a produção das seqüências sem que para isso qualquer criança tenha utilizado estratégias de nomeação. Os dados sugerem, segundo os autores, que a transitividade pode ser inferida na ausência da mediação verbal. Entretanto, os autores não descrevem como isso ocorreu nem qual medida utilizaram para essa explicação.

Assis e Sampaio (2003) planejaram duas condições experimentais, com o objetivo de explicitar os efeitos do ensino por encadeamento e justaposição de estímulos com dois conjuntos de estímulos (desenhos de aves e mamíferos) sobre o desempenho de alunos com necessidades educacionais especiais. Os resultados mostraram diferenças relevantes entre os dois grupos. Apenas um participante do Grupo 1 apresentou os desempenhos esperados após a segunda exposição aos testes. Os três participantes do Grupo 2 responderam prontamente. A familiaridade dos estímulos, no entanto, pode ter sido um fator que contribuiu para esses resultados.

O presente estudo utilizou formas abstratas para eliminar o efeito da familiaridade dos estímulos e ensinar aos portadores de necessidades educacionais especiais o comportamento de ordenar. Duas táticas de ensino foram programadas para avaliar o efeito dos procedimentos sobre o desempenho: ensino por encadeamento e por justaposição de estímulos.

\section{MÉTODO}

\section{Participantes}

A Tabela 1 resume as características relevantes dos alunos selecionados. A seleção dos alunos foi realizada por meio de contatos pessoais em sla de aula, sob a supervisão da professora. Os pais ou responsáveis eram informados sobre a pesquisa e assinaram um termo de compromisso conforme modelo da Comissão de Ética do Conselho Nacional de Saúde (Resolução 196/96).

Os participantes foram distribuídos aleatoriamente em dois grupos experimentais: Grupo "A" e Grupo "B". A designação de cada participante, por questões éticas, convencionou-se fazer com a letra $P$ (participante) seguida por um número, definido pela ordem em que o participante iniciou a atividade no estudo.

Tabela 1. Características relevantes dos participantes dos dois grupos experimentais

\begin{tabular}{|c|c|c|c|c|c|c|c|c|c|c|}
\hline & Grupo & Part. & Sexo & Idade & PPVT - R & Diagnóstico & Escolaridade & $\begin{array}{l}\text { Habilidades } \\
\text { Lingüísticas }\end{array}$ & Repertório Acadêmico & $\begin{array}{c}\text { Atividades } \\
\text { diárias }\end{array}$ \\
\hline \multirow{6}{*}{ : } & \multirow{6}{*}{ “A” } & P1 & $\mathrm{F}$ & 20,1 & 4,4 & $\begin{array}{l}\text { Dificuldade de } \\
\text { aprendizagem }\end{array}$ & $\begin{array}{c}\text { Educação de jovens } \\
\text { e adultos }\end{array}$ & $\begin{array}{l}\text { Linguagem expressiva } \\
\text { bastante comprometida }\end{array}$ & - & Dependente \\
\hline & & $\mathrm{P} 2$ & F & 17,5 & 3,7 & $\begin{array}{l}\text { Síndrome de } \\
\text { Down }\end{array}$ & $\begin{array}{c}\text { Educação de jovens } \\
\text { e adultos }\end{array}$ & $\begin{array}{c}\text { Mantém conversação } \\
\text { simples }\end{array}$ & - & Dependente \\
\hline & & P3 & F & 40,1 & 9,3 & $\begin{array}{l}\text { Dificuldade de } \\
\text { aprendizagem }\end{array}$ & $\begin{array}{c}\text { Educação de jovens } \\
\text { e adultos }\end{array}$ & $\begin{array}{l}\text { Mantém conversação } \\
\text { sofisticada }\end{array}$ & $\begin{array}{l}\text { Discrimina quantidade, cores, } \\
\text { formas, tamanhos. }\end{array}$ & Independente \\
\hline & & P7 & M & 24,1 & 6,3 & $\begin{array}{l}\text { Dificuldade de } \\
\text { aprendizagem }\end{array}$ & $\begin{array}{l}\text { Oficinaprofissionaliz } \\
\text { ante }\end{array}$ & $\begin{array}{l}\text { Mantém conversação } \\
\text { simples }\end{array}$ & - & Independente \\
\hline & & P8 & M & 31,0 & 7,0 & $\begin{array}{l}\text { Síndrome de } \\
\text { Down }\end{array}$ & $\begin{array}{l}\text { Oficinaprofissionaliz } \\
\text { ante }\end{array}$ & $\begin{array}{l}\text { Mantém conversação } \\
\text { simples }\end{array}$ & - & Independente \\
\hline & & P9 & M & 26,5 & 3,2 & $\begin{array}{l}\text { Dificuldade de } \\
\text { aprendizagem }\end{array}$ & $\begin{array}{l}\text { Oficinaprofissionaliz } \\
\text { ante }\end{array}$ & $\begin{array}{l}\text { Mantém conversação } \\
\text { simples }\end{array}$ & - & Independente \\
\hline \multirow{6}{*}{ 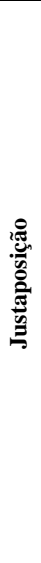 } & & $\mathrm{P} 4$ & $\mathrm{M}$ & 27,9 & 4,3 & $\begin{array}{l}\text { Síndrome de } \\
\text { Down }\end{array}$ & $\begin{array}{l}\text { Educação de jovens } \\
\text { e adultos }\end{array}$ & $\begin{array}{l}\text { Linguagem expressiva } \\
\text { bastante comprometida }\end{array}$ & - & Independente \\
\hline & & P5 & M & 17,5 & 3,7 & $\begin{array}{l}\text { Síndrome de } \\
\text { Down }\end{array}$ & $\begin{array}{c}\text { Educação de jovens } \\
\text { e adultos }\end{array}$ & $\begin{array}{l}\text { Linguagem expressiva } \\
\text { bastante comprometida }\end{array}$ & - & Dependente \\
\hline & "B" & P6 & M & 24,4 & 3,3 & $\begin{array}{l}\text { Síndrome de } \\
\text { Down }\end{array}$ & $\begin{array}{c}\text { Educação de jovens } \\
\text { e adultos }\end{array}$ & $\begin{array}{l}\text { Mantém conversação } \\
\text { simples }\end{array}$ & $\begin{array}{l}\text { Escreve o nome, identifica números e } \\
\text { letras, discrimina quantidade, cores, } \\
\text { formas, tamanhos. }\end{array}$ & Independente \\
\hline & & P10 & $\mathrm{F}$ & 21,3 & 7,0 & $\begin{array}{l}\text { Dificuldade de } \\
\text { aprendizagem }\end{array}$ & $\begin{array}{l}\text { Oficinaprofissionaliz } \\
\text { ante }\end{array}$ & $\begin{array}{c}\text { Mantém conversação } \\
\text { simples }\end{array}$ & - & Dependente \\
\hline & & P11 & F & 28,1 & 7,9 & $\begin{array}{l}\text { Síndrome } \\
\text { deDown }\end{array}$ & $\begin{array}{l}\text { Oficinaprofissionaliz } \\
\text { ante }\end{array}$ & $\begin{array}{l}\text { Mantém conversação } \\
\text { sofisticada }\end{array}$ & \multicolumn{2}{|c|}{$\begin{array}{l}\text { Escreve o nome, identifica números e Indep } \\
\text { letras, lê palavras dissílabas, realiza } \\
\text { operações de adição simples, } \\
\text { discrimina quantidade, cores, formas, } \\
\text { tamanhos. }\end{array}$} \\
\hline & & P12 & F & 20,8 & 10,9 & $\begin{array}{l}\text { Dificuldade de } \\
\text { aprendizagem }\end{array}$ & $\begin{array}{l}\text { Oficinaprofissionaliz } \\
\text { ante }\end{array}$ & $\begin{array}{l}\text { Mantém conversação } \\
\text { sofisticada }\end{array}$ & \multicolumn{2}{|c|}{$\begin{array}{l}\text { Escreve o nome, identifica números e Independente } \\
\text { letras, lê palavras dissílabas, } \\
\text { discrimina quantidade, cores, formas, } \\
\text { tamanhos. }\end{array}$} \\
\hline
\end{tabular}




\section{AMBIENTE EXPERIMENTAL E MATERIAIS}

A coleta de dados foi efetuada numa sala cedida pela Instituição, iluminada por lâmpadas fluorescentes e climatizada por um condicionador de ar. Contou-se com um microcomputador IBM PENTIUM II com a instalação de um software (REL 3.0 for Windows), especialmente desenvolvido para a pesquisa. $\mathrm{O}$ programa foi preparado para controlar e registrar os dados comportamentais, apresentar os estímulos em tentativas randomizadas e gerar relatórios das respostas apresentadas pelos participantes.

Os estímulos eram constituídos por formas abstratas, organizadas em três conjuntos denominados: "A", "B" e "C" e apresentadas no interior de "janelas" distribuídas de maneira equiidistante na tela do computador. As dimensões de cada estímulo foram proporcionalmente estabelecidas a partir da figura original no formato de $2,5 \mathrm{~cm} \times 2,5 \mathrm{~cm}$ para o primeiro estímulo de cada conjunto. Os estímulos seguintes foram reduzidos em $0,5 \mathrm{~cm}$ em altura e largura até a composição de cinco figuras por conjunto. Denominações alfanuméricas identificaram os estímulos, como mostra a Figura 1.

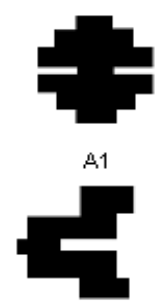

B1

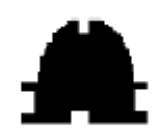

c1

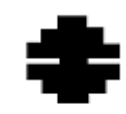

A.2

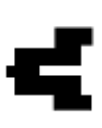

$\mathrm{B2}$

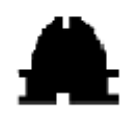

$\mathrm{C} 2$

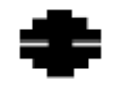

A3

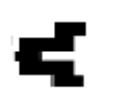

B3

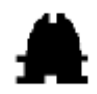

$\mathrm{C} 3$
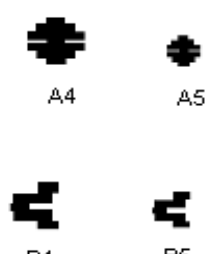

B5
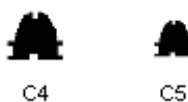

C5

Figura 1. Conjuntos de Estímulos "A", "B" e "C" utilizados no estudo.

\section{Procedimento Geral}

Um pré-teste foi aplicado inicialmente para avaliar quais alunos poderiam participar do estudo. A seleção dos participantes foi efetuada a partir de observações em sala de aula, mediante entrevistas com professores, pais ou responsáveis e através da aplicação do Peabody Picture Vocabulary Test Revised (Dunn \& Dunn, 1981), para estabelecer a idade equivalente ${ }^{2}$. Nessa ocasião um termo de

A idade equivalente resulta da comparação entre as pontuações alcançadas pelo avaliado e as pontuações de um outro individuo que participou do processo de padronização do teste. Constitui-se num indício de que os

compromisso foi assinado em cumprimento à exigência do Conselho Nacional de Saúde (Resolução 196/96).

Foram utilizados três conjuntos de estímulos, identificados como "A", "B" e "C". Os estímulos eram apresentados na tela do computador, dividida em duas áreas principais. $\mathrm{Na}$ parte superior da tela oito "janelas", dispostas lado a lado, constituíram a "área de construção" para onde eram deslocados os estímulos. Na parte inferior quatro "janelas" sobrepostas a outras quatro constituíram a "área de escolha", onde os estímulos eram inicialmente exibidos. Acima dessas áreas havia uma "janela" com a palavra "Toque" sinalizando o início de cada tentativa. Um toque do participante nessa "janela" disponibilizava os estímulos na "área de escolha", numa tentativa de ensino ou teste. A tarefa do participante era selecionar os estímulos do maior para o menor. Tocar o estímulo deslocava-o para a "área de construção", onde permanecia até que todos os demais fossem deslocados e a sequiência completada, constituindo uma tentativa.

As tentativas eram seguidas por sons de aplausos e/ou elogios e pela exibição de figuras de animais e flores, sinalizando seqüências escolhidas corretamente. A experimentadora também se referia a essas respostas com elogios.

Dois procedimentos de ensino foram programados: encadeamento e justaposição de estímulos, ambos para a formação de três sequiências de cinco estímulos. Todos os participantes foram expostos aos dois procedimentos divididos em dois grupos experimentais. Os participantes do Grupo "A" foram expostos, inicialmente, ao encadeamento e, em seguida, ao procedimento por justaposição de estímulos. O Grupo "B" iniciou pelo procedimento de justaposição de estímulos, passando em seguida ao procedimento de ensino por encadeamento. $\mathrm{O}$ delineamento experimental é apresentado na Tabela 2. Os procedimentos foram planejados em sete fases e as fases 1,2 e 5 apresentaram oito passos com as relações ensinadas gradativamente.

A Fase 1 estava programada com oito passos e apresentava as relações ensinadas entre os elementos do Conjunto "A".

A Fase 2 também estava programada com oito passos e apresentava as relações ensinadas entre os elementos do Conjunto "B".

As Fases 3 e 4 apresentaram a revisão da linha de base das relações entre os estímulos do Conjunto "A"

dois indivíduos compartilham a mesma idade de vocabulário, o que não significa que eles tenham a mesma idade cronológica ou mental. 
e "B" e os testes de substituibilidade das relações "AB" e "BA".

$\mathrm{Na}$ Fase 5 também estava programada com oito passos e apresentava as relações ensinadas entre os elementos do Conjunto "C".
As Fases 6 e 7 apresentaram a revisão da linha de base das relações entre os elementos do Conjunto "A" e "C" e testes de substituibilidade dos estímulos entre os membros dos dois conjuntos AC e CA.

Tabela 2. Delineamento Experimental dos treinos e testes programados para as duas condições

\begin{tabular}{|c|c|c|c|c|}
\hline Fases/ Passos & Tipo de Tentativa & Encadeamento & Justaposição & $\begin{array}{c}\text { Probabilidade de } \\
\text { Reforços }\end{array}$ \\
\hline Fase 1 & Treino do Conjunto"A" & & & \\
\hline Passo 01 & Treino & $(\mathrm{A} 1 \rightarrow \mathrm{A} 2)$ & $(\mathrm{A} 1 \rightarrow \mathrm{A} 2)$ & $1 / 1$ \\
\hline 02 & Sonda & $(\mathrm{A} 1 \rightarrow \mathrm{A} 2)$ & $(\mathrm{A} 1 \rightarrow \mathrm{A} 2)$ & - \\
\hline 03 & Treino & $(\mathrm{A} 1 \rightarrow \mathrm{A} 2 \rightarrow \mathrm{A} 3)$ & $(\mathrm{A} 2 \rightarrow \mathrm{A} 3)$ & $1 / 1$ \\
\hline 04 & Sonda & $(\mathrm{A} 1 \rightarrow \mathrm{A} 2 \rightarrow \mathrm{A} 3)$ & $(\mathrm{A} 1 \rightarrow \mathrm{A} 2 \rightarrow \mathrm{A} 3)$ & - \\
\hline 05 & Treino & $(\mathrm{A} 1 \rightarrow \mathrm{A} 2 \rightarrow \mathrm{A} 3 \rightarrow \mathrm{A} 4)$ & $(\mathrm{A} 3 \rightarrow \mathrm{A} 4)$ & $1 / 1$ \\
\hline 06 & Sonda & $(\mathrm{A} 1 \rightarrow \mathrm{A} 2 \rightarrow \mathrm{A} 3 \rightarrow \mathrm{A} 4)$ & $(\mathrm{A} 1 \rightarrow \mathrm{A} 2 \rightarrow \mathrm{A} 3 \rightarrow \mathrm{A} 4)$ & - \\
\hline 07 & Treino & $(\mathrm{A} 1 \rightarrow \mathrm{A} 2 \rightarrow \mathrm{A} 3 \rightarrow \mathrm{A} 4 \rightarrow \mathrm{A} 5)$ & $(\mathrm{A} 4 \rightarrow \mathrm{A} 5)$ & $1 / 1$ \\
\hline 08 & Sonda & $(\mathrm{A} 1 \rightarrow \mathrm{A} 2 \rightarrow \mathrm{A} 3 \rightarrow \mathrm{A} 4 \rightarrow \mathrm{A} 5)$ & $(\mathrm{A} 1 \rightarrow \mathrm{A} 2 \rightarrow \mathrm{A} 3 \rightarrow \mathrm{A} 4 \rightarrow \mathrm{A} 5)$ & - \\
\hline Fase 2 & Treino do Conjunto "B" & & & \\
\hline Passo 1 & Treino & $(\mathrm{B} 1 \rightarrow \mathrm{B} 2$ & $(\mathrm{B} 1 \rightarrow \mathrm{B} 2)$ & $1 / 1$ \\
\hline 02 & Sonda & $(\mathrm{B} 1 \rightarrow \mathrm{B} 2)$ & $(\mathrm{B} 1 \rightarrow \mathrm{B} 2)$ & - \\
\hline 03 & Treino & $(\mathrm{B} 1 \rightarrow \mathrm{B} 2 \rightarrow \mathrm{B} 3)$ & $(\mathrm{B} 2 \rightarrow \mathrm{B} 3)$ & $1 / 1$ \\
\hline 04 & Sonda & $(\mathrm{B} 1 \rightarrow \mathrm{B} 2 \rightarrow \mathrm{B} 3)$ & $(\mathrm{B} 1 \rightarrow \mathrm{B} 2 \rightarrow \mathrm{B} 3)$ & - \\
\hline 05 & Treino & $(\mathrm{B} 1 \rightarrow \mathrm{B} 2 \rightarrow \mathrm{B} 3 \rightarrow \mathrm{B} 4)$ & $(\mathrm{B} 3 \rightarrow \mathrm{B} 4)$ & $1 / 1$ \\
\hline 06 & Sonda & $(\mathrm{B} 1 \rightarrow \mathrm{B} 2 \rightarrow \mathrm{B} 3 \rightarrow \mathrm{B} 4)$ & $(\mathrm{B} 1 \rightarrow \mathrm{B} 2 \rightarrow \mathrm{B} 3 \rightarrow \mathrm{B} 4)$ & - \\
\hline 07 & Treino & $(\mathrm{B} 1 \rightarrow \mathrm{B} 2 \rightarrow \mathrm{B} 3 \rightarrow \mathrm{B} 4 \rightarrow \mathrm{B} 5)$ & $(\mathrm{B} 4 \rightarrow \mathrm{B} 5)$ & $1 / 1$ \\
\hline 08 & Sonda & $(\mathrm{B} 1 \rightarrow \mathrm{B} 2 \rightarrow \mathrm{B} 3 \rightarrow \mathrm{B} 4 \rightarrow \mathrm{B} 5)$ & $(\mathrm{B} 1 \rightarrow \mathrm{B} 2 \rightarrow \mathrm{B} 3 \rightarrow \mathrm{B} 4 \rightarrow \mathrm{B} 5)$ & - \\
\hline \multirow[t]{2}{*}{ Fase 3} & Revisão Conjunto "A" & $(\mathrm{A} 1 \rightarrow \mathrm{A} 2 \rightarrow \mathrm{A} 3 \rightarrow \mathrm{A} 4 \rightarrow \mathrm{A} 5)$ & $(\mathrm{A} 1 \rightarrow \mathrm{A} 2)(\mathrm{A} 2 \rightarrow \mathrm{A} 3)(\mathrm{A} 3 \rightarrow \mathrm{A} 4)(\mathrm{A} 4 \rightarrow \mathrm{A} 5)$ & $1 / 1$ \\
\hline & Revisão Conjunto "B" & $(\mathrm{B} 1 \rightarrow \mathrm{B} 2 \rightarrow \mathrm{B} 3 \rightarrow \mathrm{B} 4 \rightarrow \mathrm{B} 5)$ & $(\mathrm{B} 1 \rightarrow \mathrm{B} 2)(\mathrm{B} 2 \rightarrow \mathrm{B} 3)(\mathrm{B} 3 \rightarrow \mathrm{B} 4)(\mathrm{B} 4 \rightarrow \mathrm{B} 5)$ & $1 / 1$ \\
\hline \multirow[t]{2}{*}{ Fase 4} & Teste AB & $(\mathrm{A} 1 \rightarrow \mathrm{B} 2 \rightarrow \mathrm{A} 3 \rightarrow \mathrm{B} 4 \rightarrow \mathrm{A} 5)$ & $(\mathrm{A} 1 \rightarrow \mathrm{B} 2 \rightarrow \mathrm{A} 3 \rightarrow \mathrm{B} 4 \rightarrow \mathrm{A} 5)$ & - \\
\hline & Teste BA & $(\mathrm{B} 1 \rightarrow \mathrm{A} 2 \rightarrow \mathrm{B} 3 \rightarrow \mathrm{A} 4 \rightarrow \mathrm{B} 5)$ & $(\mathrm{B} 1 \rightarrow \mathrm{A} 2 \rightarrow \mathrm{B} 3 \rightarrow \mathrm{A} 4 \rightarrow \mathrm{B} 5)$ & - \\
\hline Fase 5 & Treino do Conjunto"C" & & & \\
\hline Passo 1 & Treino & $(\mathrm{C} 1 \rightarrow \mathrm{C} 2$ & $(\mathrm{C} 1 \rightarrow \mathrm{C} 2)$ & $1 / 1$ \\
\hline 02 & Sonda & $(\mathrm{C} 1 \rightarrow \mathrm{C} 2)$ & $(\mathrm{C} 1 \rightarrow \mathrm{C} 2)$ & - \\
\hline 03 & Treino & $(\mathrm{C} 1 \rightarrow \mathrm{C} 2 \rightarrow \mathrm{C} 3)$ & $(\mathrm{C} 2 \rightarrow \mathrm{C} 3)$ & $1 / 1$ \\
\hline 04 & Sonda & $(\mathrm{C} 1 \rightarrow \mathrm{C} 2 \rightarrow \mathrm{C} 3)$ & $(\mathrm{C} 1 \rightarrow \mathrm{C} 2 \rightarrow \mathrm{C} 3)$ & - \\
\hline 05 & Treino & $(\mathrm{C} 1 \rightarrow \mathrm{C} 2 \rightarrow \mathrm{C} 3 \rightarrow \mathrm{C} 4)$ & $(\mathrm{C} 3 \rightarrow \mathrm{C} 4)$ & $1 / 1$ \\
\hline 06 & Sonda & $(\mathrm{C} 1 \rightarrow \mathrm{C} 2 \rightarrow \mathrm{C} 3 \rightarrow \mathrm{C} 4)$ & $(\mathrm{C} 1 \rightarrow \mathrm{C} 2 \rightarrow \mathrm{C} 3 \rightarrow \mathrm{C} 4)$ & - \\
\hline 07 & Treino & $(\mathrm{C} 1 \rightarrow \mathrm{C} 2 \rightarrow \mathrm{C} 3 \rightarrow \mathrm{C} 4 \rightarrow \mathrm{C} 5)$ & $(\mathrm{C} 4 \rightarrow \mathrm{C} 5)$ & $1 / 1$ \\
\hline 08 & Sonda & $(\mathrm{C} 1 \rightarrow \mathrm{C} 2 \rightarrow \mathrm{C} 3 \rightarrow \mathrm{C} 4 \rightarrow \mathrm{C} 5)$ & $(\mathrm{C} 1 \rightarrow \mathrm{C} 2 \rightarrow \mathrm{C} 3 \rightarrow \mathrm{C} 4 \rightarrow \mathrm{C} 5)$ & - \\
\hline \multirow[t]{2}{*}{ Fase 6} & Revisão Conjunto "A" & $(\mathrm{A} 1 \rightarrow \mathrm{A} 2 \rightarrow \mathrm{A} 3 \rightarrow \mathrm{A} 4 \rightarrow \mathrm{A} 5)$ & $(\mathrm{A} 1 \rightarrow \mathrm{A} 2)(\mathrm{A} 2 \rightarrow \mathrm{A} 3)(\mathrm{A} 3 \rightarrow \mathrm{A} 4)(\mathrm{A} 4 \rightarrow \mathrm{A} 5)$ & $1 / 1$ \\
\hline & Revisão Conjunto "C" & $(\mathrm{C} 1 \rightarrow \mathrm{C} 2 \rightarrow \mathrm{C} 3 \rightarrow \mathrm{C} 4 \rightarrow \mathrm{C} 5)$ & $(\mathrm{C} 1 \rightarrow \mathrm{C} 2)(\mathrm{C} 2 \rightarrow \mathrm{C} 3)(\mathrm{C} 3 \rightarrow \mathrm{C} 4)(\mathrm{C} 4 \rightarrow \mathrm{C} 5)$ & $1 / 1$ \\
\hline \multirow[t]{2}{*}{ Fase 7} & Teste AC & $(\mathrm{A} 1 \rightarrow \mathrm{C} 2 \rightarrow \mathrm{A} 3 \rightarrow \mathrm{C} 4 \rightarrow \mathrm{A} 5)$ & $(\mathrm{A} 1 \rightarrow \mathrm{C} 2 \rightarrow \mathrm{A} 3 \rightarrow \mathrm{C} 4 \rightarrow \mathrm{A} 5)$ & - \\
\hline & Teste CA & $(\mathrm{C} 1 \rightarrow \mathrm{A} 2 \rightarrow \mathrm{C} 3 \rightarrow \mathrm{A} 4 \rightarrow \mathrm{C} 5)$ & $(\mathrm{C} 1 \rightarrow \mathrm{A} 2 \rightarrow \mathrm{C} 3 \rightarrow \mathrm{A} 4 \rightarrow \mathrm{C} 5)$ & - \\
\hline
\end{tabular}

Instruções mínimas eram adotadas durante o estudo. Por exemplo, durante o ensino da tarefa, a experimentadora dizia: "Toque na figura" ou "Toque em uma figura e depois em outra". Durante os testes, a experimentadora dizia: "Agora não haverá aplausos nem desenhos na tela, mas você deverá continuar tocando na tela até terminar, entendeu ?" O critério de acerto era de três tentativas consecutivas, sem erro. Após cada sequiência correta, uma animação gráfica e um som de aplausos eras apresentados na tela e a experimentadora elogiava: "muito bem!", "legal”, "certo". Respostas incorretas produziam uma nova configuração de estímulos ${ }^{3}$ após intervalo de $1 \mathrm{~s}$ (intervalo entre tentativas). No final de cada sessão, a experimentadora oferecia um "brinde" ao participante, independentemente do seu desempenho. Caso houvesse disponibilidade do participante, uma outra sessão era iniciada no

3 Entende-se por configuração a apresentação aleatória dos estímulos no painel de escolha. 
mesmo dia, com intervalo de pelo menos 10 minutos. No ensino por encadeamento, o número de tentativas era de 10 vezes, no máximo, independentemente do número de estímulos presente na seqüência (dois, três, quatro ou cinco). No ensino por justaposição de estímulos apresentavam-se quatro tentativas, no máximo.

\section{ENSINO POR ENCADEAMENTO}

No encadeamento, as tentativas foram planejadas com configurações que introduziram os estímulos de forma gradual até que todos fossem apresentados simultaneamente na "área de escolha".

$\mathrm{Na}$ Fase 1, um toque do participante na "janela" superior produzia o aparecimento de um estímulo na "área de escolha". Um toque no estímulo deslocava-o para a "área de construção". Essa tentativa inicial apresentava uma única alternativa de escolha, garantindo ao participante ter sua escolha reforçada.

Em seguida, uma nova configuração de estímulos era exibida. Dois estímulos da mesma forma e tamanhos diferentes eram apresentados na "área de escolha”. Era requerido ao participante escolher um e depois outro estímulo, e sua resposta era reforçada no final da seqüência sempre que selecionava primeiro o maior e depois o menor, e assim sucessivamente, até completar a seqüência de cinco estímulos $(\mathrm{A} 1 \rightarrow \mathrm{A} 2 \rightarrow \mathrm{A} 3 \rightarrow \mathrm{A} 4 \rightarrow \mathrm{A} 5)$. O procedimento de ensino adotou a formação de uma cadeia para frente, na qual apenas a última resposta era seguida pelo feedback.

Ensino por justaposição de estímulos

Nesta tática de ensino, as tentativas eram apresentadas em configurações que exibiam apenas dois estímulos na "área de escolha".

Um toque exibia duas figuras da mesma forma em tamanhos diferentes. A resposta requerida era tocar uma e depois a outra, e o feedback era apresentado para a seleção da maior para a menor, estabelecendose a relação $\mathrm{B} 1 \rightarrow \mathrm{B} 2$. Em um novo arranjo, a primeira figura do par era excluída, a segunda era mantida e um novo estímulo era introduzido, constituindo-se um novo par de estímulos. A tarefa, novamente, era selecionar a maior e depois a menor, estabelecendo-se a relação $\mathrm{B} 2 \rightarrow \mathrm{B} 3 \mathrm{e}$, assim, sucessivamente até completar a relação B4 $\rightarrow$ B5.

\section{Sondas}

Todas as relações ensinadas eram seguidas de tentativas que avaliavam a produção de seqüências com dois, três, quatro e cinco estímulos, em extinção.
As sondas eram aplicadas em tentativas únicas, sempre após uma relação ensinada.

O procedimento de ensino por encadeamento apresentava tentativas de sondas com dois, três, quatro ou cinco estímulos. No procedimento por justaposição de estímulos as tentativas de sondas eram sempre com dois estímulos, visto que as configurações de estímulos no ensino apresentavam apenas dois estímulos.

\section{Testes}

Os testes de substituibilidade (conectividade) avaliaram a intercambialidade funcional entre os membros de diferentes conjuntos de estímulos. Propriedades de relações entre estímulos em seqüência como a transitividade, estão implícitas nessas relações mais complexas de conectividade. Caso o participante não respondesse corretamente na primeira tentativa, a mesma configuração de estímulos era reapresentada mais uma vez. Esses testes apresentavam tentativas que exibiam estímulos de dois conjuntos "A" e "B" ou "A" e "C". A tarefa era selecionar os estímulos pelo tamanho do maior para o menor, na ordem em que eram apresentados no ensino. Nas tentativas da relação $\mathrm{AB}$, o primeiro estímulo selecionado devia ser A1 seguido por B2, A3, B4 e A5. Para o teste BA, a primeira seleção devia ser B1 seguida por A2, B3, A4 e B5. Esses arranjos avaliavam se os estímulos dos dois conjuntos ensinados eram substituíveis (equivalentes) entre si e se podiam, então, exercer as mesmas funções de ordem nos dois conjuntos, caracterizando a formação de classes ordinais.

\section{RESULTADOS}

\section{Relações ensinadas}

Todos os participantes alcançaram o critério de acerto durante o treino. P1, P2 e P3 precisaram de cinco exposições à seqüência mais longa, com cinco estímulos do Conjunto A. P7 respondeu prontamente (na primeira tentativa) a todas as seqüências previstas para este grupo. P8 e P9 precisaram de sete exposições na fase com a sequiência mais longa,com cinco estímulos no conjunto $\mathrm{A}$, e precisaram de cinco exposições quando expostos ao conjunto de estímulos B.

$\mathrm{Na}$ outra condição experimental programada para o Grupo A, todos os participantes responderam consistentemente com a literatura, conforme pode ser observado na Tabela 3. 
Tabela 3. Número de respostas corretas em cada seqüência com dois, três, quatro e cinco estímulos nos três conjuntos "A", "B" e "C" por participante no Grupo "A"

\begin{tabular}{|c|c|c|c|c|c|c|c|c|c|c|c|c|c|c|c|c|c|c|c|c|c|c|c|c|c|}
\hline & \multicolumn{12}{|c|}{ Encadeamento } & \multicolumn{12}{|c|}{ Justaposição } \\
\hline & & \multicolumn{4}{|c|}{ Conjunto "A" } & \multicolumn{4}{|c|}{ Conjunto 'B"' } & \multicolumn{4}{|c|}{ Conjunto “C”' } & \multicolumn{4}{|c|}{ Conjunto "A" } & \multicolumn{4}{|c|}{ Conjunto "B"' } & \multicolumn{4}{|c|}{ Conjunto “C”' } \\
\hline & & \multicolumn{4}{|c|}{ Número de Estímulos } & \multicolumn{4}{|c|}{ Número de Estímulos } & \multicolumn{4}{|c|}{ Número de Estímulos } & \multicolumn{4}{|c|}{ Número de Estímulos } & \multicolumn{4}{|c|}{ Número de Estímulos } & \multicolumn{4}{|c|}{ Número de Estímulos } \\
\hline & & Dois & s Três & Quatro & Cinco & Dois & s Três & Quatro & Cinco & Dois & s Três & Quatro & Cinco & Dois & Dois & Dois & Dois & Dois & Dois & Dois & Dois & Dois & Dois & Dois & Dois \\
\hline \multirow{6}{*}{ 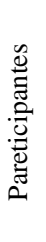 } & $\mathrm{P} 1$ & $*$ & $*$ & $*$ & $* *$ & $*$ & $*$ & $*$ & $*$ & $*$ & $*$ & $*$ & $*$ & $*$ & $*$ & $*$ & $*$ & $*$ & $*$ & $*$ & $*$ & $*$ & $*$ & $*$ & $*$ \\
\hline & $\mathrm{P} 2$ & $*$ & $*$ & $*$ & $* *$ & $*$ & $*$ & $*$ & $*$ & $*$ & $*$ & $*$ & $*$ & $*$ & $*$ & $*$ & $*$ & $*$ & $*$ & $*$ & $*$ & $*$ & $*$ & $*$ & $*$ \\
\hline & P3 & $*$ & $*$ & $*$ & $* * *$ & $*$ & $*$ & $*$ & $*$ & $*$ & $*$ & $*$ & $*$ & $*$ & $*$ & $*$ & $*$ & $*$ & $*$ & $*$ & $*$ & $*$ & $*$ & $*$ & $*$ \\
\hline & $\mathrm{P} 7$ & $*$ & $*$ & $*$ & $*$ & $*$ & $*$ & $*$ & $*$ & $*$ & $*$ & $*$ & $*$ & $*$ & $*$ & $*$ & $*$ & $*$ & $*$ & $*$ & $*$ & $*$ & $*$ & $*$ & $*$ \\
\hline & P8 & $*$ & $*$ & $*$ & $* * *$ & $*$ & $*$ & $*$ & $* *$ & $*$ & $*$ & $*$ & $*$ & $*$ & $*$ & $*$ & $*$ & $*$ & $*$ & $*$ & $*$ & $*$ & $*$ & $*$ & $*$ \\
\hline & P9 & $*$ & $*$ & $*$ & $* * *$ & $*$ & $*$ & $*$ & $* *$ & $*$ & $*$ & $*$ & $*$ & $*$ & $*$ & $*$ & $*$ & $*$ & $*$ & $*$ & $*$ & $*$ & $*$ & $*$ & $*$ \\
\hline
\end{tabular}

*O participante alcançou o critério de acerto nas primeiras três tentativas consecutivas sem erro;

** O participante precisou de cinco exposições;

*** O participante precisou de sete exposições.

A Tabela 4 apresenta o número de respostas corretas dos participantes no Grupo B. Na condição de justaposição P4, P5, P10 e P12 precisaram de cinco exposições ao conjunto de estímulos A e P4, P5 e P6 precisaram de cinco exposições ao conjunto $\mathrm{B}$. $\mathrm{Na}$ tática por encadeamento para ordenar os três conjuntos de estímulos, os participantes $\mathrm{P} 4, \mathrm{P} 5$ e P6 precisaram de cinco exposições para completar a seqüência de cinco estímulos do conjunto A.

Tabela 4. Número de respostas corretas em cada sequiência com dois, três, quatro e cinco estímulos nos três conjuntos "A", "B" e "C" por participante no Grupo "B"

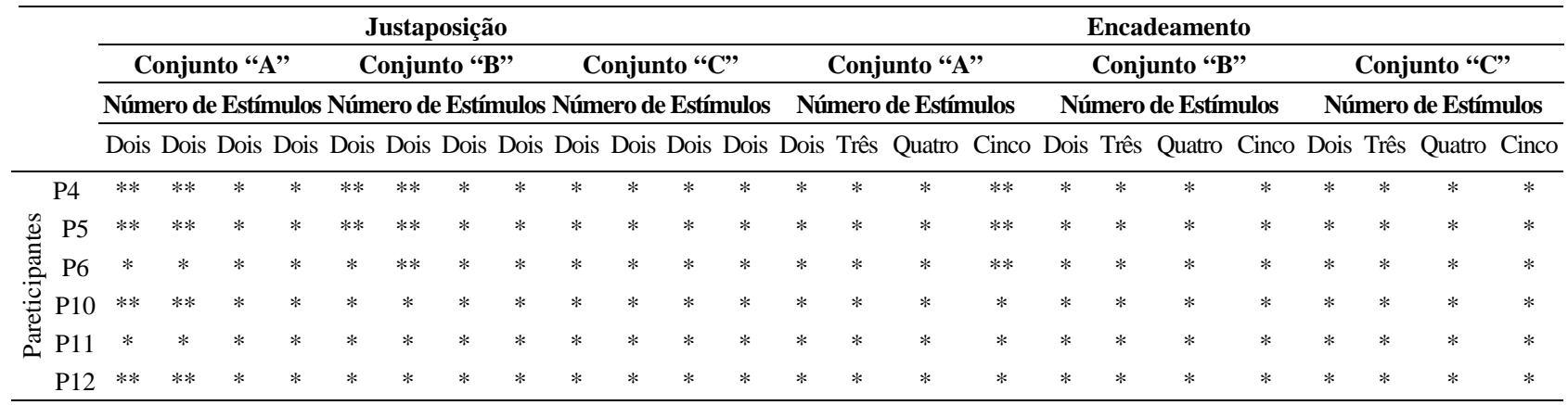

*O participante alcançou o critério de acerto nas primeiras três tentativas consecutivas sem erro;

** O participante precisou de cinco exposições.

A Tabela 5 apresenta os resultados dos testes de substituibilidade $\mathrm{AB}, \mathrm{BA}, \mathrm{AC}$ e $\mathrm{CA}$ dos dois grupos experimentais.

Tabela 5. Desempenho dos participantes dos dois grupos experimentais no teste de substituibilidade (conectividade)

\begin{tabular}{lcccccccccccc}
\hline & \multicolumn{10}{c}{ Relações } & \multicolumn{1}{c}{ Grupo A } & \multicolumn{1}{c}{ Grupo B } \\
\cline { 2 - 11 } testadas & P1 & P2 & P3 & P7 & P8 & P9 & P4 & P5 & P6 & P10 & P11 & P12 \\
\hline AB & C & IC & C & C & C & C & C & I & IC & C & C & C \\
BA & C & IC & C & C & C & C & IC & - & II & C & C & C \\
AC & C & C & C & C & C & C & - & - & - & C & C & C \\
CA & C & C & C & C & C & C & - & - & - & C & C & C \\
AB & C & C & C & II & C & C & C & II & C & C & C & C \\
BA & C & C & IC & C & C & C & C & II & C & C & C & C \\
AC & C & C & C & IC & C & C & & C & II & C & C & C \\
CA & C & C & IC & II & C & C & & C & II & C & C & C \\
\hline
\end{tabular}

Nota: $\mathrm{C}=$ Desempenho correto; $\mathrm{IC}=$ Desempenho correto na segunda tentativa; $\mathrm{Il}$ = Desempenho incorreto; - = O participante não foi exposto ao teste.
No Grupo A o participante P2 respondeu corretamente aos testes $\mathrm{AB}$ e $\mathrm{BA}$ na primeira condição experimental com atraso, ou seja, na segunda tentativa. Os demais participantes responderam prontamente. $\mathrm{Na}$ segunda condição experimental deste grupo, os participantes P3 e P7 responderam com atraso ou responderam incorretamente aos testes. Os demais participantes responderam corretamente.

No Grupo B os participantes P4, P5 e P6 responderam corretamente, mas com atraso, alguns testes, ou responderam incorretamente (ver Tabela 5).; porém não foram expostos aos testes ( $\mathrm{AC}$ e $\mathrm{CA}$ ), por não terem alcançado o critério estabelecido para a linha de base na fase de revisão (ver Tabela 2). Os demais participantes responderam corretamente na primeira condição. 
$\mathrm{Na}$ segunda condição, um participante (P5) respondeu incorretamente a todos os testes. Os demais participantes responderam corretamente na primeira tentativa.

\section{DISCUSSÃO}

O presente trabalho buscou verificar o efeito de dois procedimentos de ensino sobre o comportamento de ordenar formas abstratas em portadores de necessidades educacionais especiais. As duas táticas de ensino (encadeamento e justaposição de estímulos) foram efetivas na instalação dos repertórios planejados, confirmando achados anteriores (Assis \& Sampaio, 2003). Isso ficou evidenciado nos desempenhos apresentados por nove participantes expostos à primeira parte da condição experimental programada para cada um dos dois grupos experimentais. Todos os participantes demonstraram desempenhos consistentes com a literatura no treino com ambos os procedimentos de ensino. Entretanto, alguns precisaram de mais reexposições nas seqüências mais longas, quando expostos ao primeiro conjunto de estímulos "A".

$\mathrm{Na}$ continuidade da condição experimental prevista para cada grupo, o ensino por encadeamento mostrou-se eficaz para recuperar os desempenhos de dois participantes (P4 e P6) do segundo grupo que não haviam ordenado os estímulos nos testes AC e CA. O terceiro participante (P5) apresentou um padrão sistemático de respostas incompatíveis com o desempenho planejado. Ele separou os estímulos em subconjuntos ordenados dentro de uma mesma seqüência, seguindo a ordem em que os estímulos foram apresentados nas tentativas de ensino (por exemplo, A1, A3, A5, B2, B4). Esse padrão também foi observado no único participante (P7) que não apresentou os resultados previstos quando foi exposto à tática de justaposição de estímulos, na condição experimental programada para o Grupo A. Stromer e cols. (1993) observaram padrão semelhante no desempenho de um participante adulto normal, mas não esclareceram o que controlou as respostas. Embora essas respostas não tenham sido especificadas nas contingências de reforçamento programadas, não há dúvida de que as discriminações necessárias para o desempenho programado foram estabelecidas.

Para o estudo aqui descrito, duas dimensões do estímulo foram críticas no estabelecimento dos repertórios planejados: o tamanho dos estímulos, que variou em cinco níveis, com reduções proporcionais (do maior ao menor), e a posição que cada estímulo ocupou na seqüência ensinada, delineada nas duas táticas de ensino, cujo requerimento da tarefa exigiu o agrupamento dos estímulos por atributos que caracterizam conceitos de tamanho (maior, menor).

Uma terceira dimensão que pode ter sido responsável pelo padrão descrito anteriormente foi a forma dos estímulos. Os estímulos foram agrupados pela similaridade física que existia entre eles. A aplicação de testes que incluíssem tentativas com um estímulo apenas de cada conjunto poderia ter controlado esta variável. Neste caso, cada conjunto poderia ter sido composto por três membros, cujos tamanhos estariam mais evidenciados, além de evitar a repetição de estímulos do mesmo conjunto (Galy \& cols., 2003).

O procedimento que utilizou o encadeamento mostrou-se superior na instalação, manutenção e recuperação de repertórios deteriorados, em relação ao procedimento que adotou a justaposição de estímulos. A característica principal observada na tática de encadeamento está evidenciada na Tabela 2: a exposição continuada do estímulo em todas as tentativas de ensino até que o critério de acerto fosse alcançado. Na segunda tática que adotou a justaposição de estímulos, os estímulos iniciais eram excluídos da configuração e novos estímulos eram introduzidos, de maneira que nunca aparecessem todos simultaneamente. No entanto, as relações derivadas dessa tática, segundo Stromer e cols. (1993), são verdadeiramente transitivas, justamente porque os estímulos que formam a base para os testes de substituibilidade nunca aparecem juntos nas tentativas de ensino.

Outro fator que pode ter contribuído para o insucesso dos três participantes que foram submetidos, inicialmente, ao procedimento de justaposição de estímulos está relacionado com a exigência da tarefa experimental, o que nos remete para as variáveis de sujeitos. Nas tentativas de ensino, a tarefa foi tocar duas figuras em ordem decrescente de tamanho. As sondas aplicadas após cada relação ensinada exigiam do participante responder a todos estímulos, inclusive àqueles excluídos dos pares anteriores (ver Tabela 2). Considerando-se que esses participantes (P4, P5 e P6) apresentaram idade equivalente abaixo de cinco anos (ver Tabela 1), pode-se supor que o baixo nível de vocabulário tenha influenciado o desempenho.

Holcomb e cols. (1997) atribuíram os resultados positivos obtidos em relações transitivas por crianças jovens normais à coerência no formato de treino e testes. Ou seja, uma outra possibilidade para esses resultados negativos talvez esteja no estabelecimento, 
durante o treino, de topografias de controle de estímulos deficiente (McIlvane, Serna, Dube \& Stromer, 2000). Ou seja, todas as discriminações devem ser verificadas e o participante deve responder às diferentes configurações de estímulo antes dos testes. De fato, a proposta teórica da coerência da topografia de controle de estímulos explica que pequenas mudanças do procedimento de ensino para o de testes podem gerar controle por variáveis não planejadas, exigindo a necessidade de programar testes inseridos entre tentativas de linha de base. Portanto, muito provavelmente, a introdução de sondas exigindo um responder à sequiência completa no procedimento de justaposição pode ter dificultado a tarefa do participante com essa tática. As sondas deveriam apresentar o mesmo formato do treino com dois estímulos. McIlvane e cols. (2000, p. 89), afirmaram:

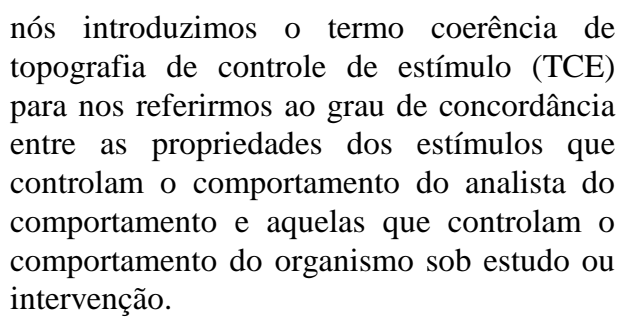

Além disso, Green e Saunders (1998, p. 253) afirmaram que "a emergência gradual durante os testes pode ser influenciada pela ordem de apresentação dos mesmos". Neste estudo, vários participantes precisaram de uma segunda tentativa de sondas e testes para responder consistentemente.

Classes de estímulos podem ser estabelecidas a partir de relações arbitrárias entre estímulos que não guardam semelhança entre si, compartilhando uma mesma função discriminativa. Para que isso seja evidenciado, é necessário verificar os efeitos de variáveis sobre um membro da classe em relação aos demais membros. Quando as funções de um membro se transferem para qualquer outro, diz-se que eles são funcionalmente equivalentes. Nas classes ordinais, as funções que se transferem são as funções de ordem (primeiro, segundo, terceiro e assim por diante) que um estímulo ocupou, sendo exercidas por outro que ocupou a mesma posição em seqüências diferentes. Assim, segundo Lima e Assis (2003, p. 82), “a verificação de classes ordinais pressupõe que todos os estímulos na classe sejam mutuamente substituíveis no controle de um mesmo desempenho, e que qualquer propriedade controladora adquirida por um membro da classe deva ser compartilhada por todos os outros membros".
Investigar a transferência de funções ordinais para novos estímulos também poderia corroborar esses resultados, tarefa que não foi possível neste estudo, ampliando consideravelmente o potencial desses procedimentos de ensino através de generalizações. Uma possibilidade que poderia vir a ser adotada seria um teste envolvendo três novos conjuntos de estímulos com formas diferentes daquelas que foram utilizadas no ensino para avaliar se o aluno ordenaria do maior para o menor. Resultados promissores foram obtidos com crianças do pré-escolar em estudos envolvendo comportamento conceitual numérico (numerosidade) em ambiente não-informatizado (Ribeiro, 2004).

Estudos ainda poderiam investigar a emergência de classes ordinais sob contingências de quatro termos, especialmente com essa população. Os resultados obtidos têm apresentado variabilidade intersujeitos (Portella, Sampaio, Baptista \& Assis, 2003). A literatura nesta área não apresentou até o presente momento resultados confiáveis com portadores de necessidades educacionais especiais.

A principal contribuição deste estudo está relacionada à identificação de uma variável de procedimento por encadeamento como relevante no ensino de relações ordinais, especialmente com portadores de necessidades educacionais especiais, que apresentam, em geral, controle restrito de estímulos. Nas relações de grandeza, que caracterizam o comportamento matemático, por exemplo, parece ser necessário que a posição do estímulo seja ensinada para que o aluno discrimine aquele que vem antes e aquele que vem depois. Com relação ao comportamento sintático, a construção da frase depende da posição que ocupam nomes, qualidades e ações ou em que são funcionalmente substituídos por seus sinônimos, sem prejuízo da localização correta. Uma tecnologia de ensino individualizada derivada de dados empíricos, como os obtidos neste estudo, poderia conduzir com sucesso à aprendizagem de relações complexas, como aquelas envolvidas nos comportamentos acima referidos.

\section{REFERÊNCIAS}

Assis, G. J. A. \& Sampaio, M. E. C. (2003). Efeitos de Dois Procedimentos de Ensino para Formação de Classes Seqüenciais. Revista Interação em Psicologia, 7(2), 52-63.

Catania, A. C. (1999, $4^{\mathrm{a}}$ ed.). Aprendizagem: comportamento, linguagem e cognição. Porto Alegre: Editora Artes Médicas (originalmente publicado em 1998). 
Dunn, L. M. \& Dunn, I. M. (1981). Peabody Picture Vocabulary Test. Circle Pines, MN: American Guidance Service.

Galy, E., Camps, J. F. \& Melan, C. (2003). Sequence Class Formation Following Learning of Short Sequences. The Psychological Record, 53, 635-645.

Green, G. \& Saunders, R. R. (1998). Stimulus equivalence. Em K. Lattal \& M. Perone (Orgs.), Handbook of Research Methods in Human Operant Behavior (pp. 229-262). New York: Plenum Press.

Green, G., Stromer, R. \& Mackay, H. (1993). Relational learning in stimulus sequences. The Psychological Record, 43, 599-616.

Holcomb, W. L., Stromer, R. \& Mackay, H. A. (1997). Transitivity and Emergent Sequence Performances in Young Children. Journal of Experimental Child Psychology, 65, 96124.

Lazar, R. (1977). Extending sequence-class membership with matching to sample. Journal of the Experimental Analysis of Behavior, 27, 381-392.

Lima, M. P. \& Assis, G. J. A. (2003). Emergência de classes seqüenciais após treino com pareamento consistente. Psicologia: Teoria e Pesquisa, 19(1), 75-84

Mackay, H. A. \& Brown, S. M. (1971). Teaching serial position sequences to monkeys with a delayed matching-tosample procedure. Journal of the Experimental Analysis of Behavior, 15, 335-345.

McIlvane, W. J., Serna, R. W., Dube, W. V. \& Stromer, R. (2000). Stimulus control topography coherente and stimulus equivalente: reconciling test outcomes with theory. Em J. C. Leslie \& D. Blackman. (Orgs.), Experimental and Applied
Analysis of Human Behavior (pp. 85-110). Reno, NV: Context Press.

Portella, I. M. V., Sampaio, M. E. C., Baptista, M. Q. G. \& Assis, G. J. A. (2003). Em busca de relações ordinais sob controle condicional em portadores de deficiência mental. Resumos de Comunicações Científicas, XII Encontro Nacional da Associação Brasileira de Psicoterapia e Medicina Comportamental (ABPMC), Londrina.

Ribeiro, M. P. L. (2004). Comportamento matemático: relações ordinais e inferência transitiva em crianças com risco psicossocial para dificuldades de aprendizagem. Tese de Doutorado Não-Publicada, apresentada ao Programa de PósGraduação em Psicologia, Universidade Federal do Espírito Santo.

Sidman, M. \& Rosenberger, P. B. (1967). Several methods for teaching serial, position sequences to monkeys. Journal of the Experimental Analysis of Behavior, 10, 467-478.

Sigurdardottir, Z. G., Green, G. \& Saunders, R. R. (1990). Equivalence classes generated by sequence training. Journal of the Experimental Analysis of Behavior, 53, 47-63.

Stevens, S. S. (1951). Mathematics, Measurement and Psychophysics. Em: S. S. Stevens (Org.), Handbook of Experimental Psychology (pp. 1-44). New York: John Wiley.

Stromer, R., Mackay, H. A., Cohen, M. \& Stoddard, L. T. (1993). Sequence learning in individuals with behavioral limitations. Journal of Intellectual Disability Research, 37, 243-261.

Recebido em 23/03/2005 Aceito em 30/09/2005
Endereço para correspondência:
Grauben José Alves de Assis, Trav. Castelo Branco, 1923/301, Bairro do Guamá, CEP 66.063.420, Belém-Pará.E-mail: gjaa@cpgp.ufpa.br 